\title{
Kossel diffraction in perfect crystals: $X$-ray standing waves in reverse
}

\author{
T. Gog, D. Bahr, and G. Materlik \\ Hamburger Synchrotronstrahlungslabor HASYLAB am Deutschen Elektronen-Synchrotron DESY, 22603 Hamburg, Germany
}

(Received 28 November 1994; revised manuscript received 10 January 1995)

\begin{abstract}
By virtue of the optical reciprocity theorem, Kossel diffraction in perfect crystals can be viewed as the reverse process of $\mathrm{x}$-ray standing waves. An experimental method can then be devised to determine atomic positions in the bulk or at a surface of a crystal by analyzing the profile of angularly resolved Kossel lines. It is demonstrated that such a technique is sensitive enough to be applied to very dilute atomic concentrations of monolayer equivalency. Experimental results and a quantitative analysis for a buried, $7 \AA$ thick layer of $\mathrm{CoSi}_{2}$ on a $\mathrm{Si}(111)$ substrate reveal the lattice position of $\mathrm{Co}$ atoms and confirm that Co-Si bonds form the interface between the $\mathrm{CoSi}_{2}$ layer and its $\mathrm{Si}$ substrate.
\end{abstract}

Kossel diffraction arises when atoms inside a crystal are excited by incident photons, electrons, protons or ions and subsequently deexcite by emitting fluorescence, thus serving as internal sources of radiation. Most of the isotropically distributed fluorescence will be reabsorbed or leave the crystal without further interaction. Some will leave the crystal after undergoing single-scattering processes, thereby providing a source for fluorescence holography. ${ }^{1,2}$ Some other fraction of it, however, will be propagating in directions where Bragg's law is satisfied for a set of diffraction planes of the surrounding lattice and for the given fluorescence energy. This fraction will then be subject to single-scattering, kinematical diffraction or to multiple-scattering, dynamical diffraction. Directions satisfying Bragg's law lie on so-called Kossel cones with opening half-angles of $\left(90^{\circ}-\Theta_{B}\right), \Theta_{B}$ being the Bragg angle, and central axes perpendicular to the diffraction planes (Fig. 1). In the case of a dynamically diffracting crystal Kossel cones will feature a radial fine structure of intensity, reminiscent of the depth of the internal source and its position within the unit cell.

Kossel diffraction was discovered experimentally ${ }^{3}$ in 1934 and subsequently explained theoretically by von Laue, ${ }^{4}$ later also by others ${ }^{5,6}$ within the framework of the dynamical theory of x-ray diffraction. The possibility of obtaining structural information from dynamical Kossel lines was pointed out $^{7}$ and an experiment has been proposed ${ }^{8,9}$ to determine the phase of structure factors in noncentrosymmetric crystals. Most recently, Kossel experiments with the aim of studying bulk structures ${ }^{10}$ and impurities in nearly perfect crystals ${ }^{11}$ have been conducted.

The focus of this article is the inherent interrelation between Kossel diffraction and x-ray standing waves (XSW), which suggests an experimental technique to determine lattice locations of impurity or overlayer atoms in perfect or nearly perfect crystals by means of angularly resolved Kossel lines. It is demonstrated that such a technique is indeed sensitive enough to handle very dilute atomic concentrations of monolayer equivalence and yields results fully equivalent to outcomes of analogously prepared XSW measurements. For a system of two atomic bilayers of $\mathrm{CoSi}_{2}$ sandwiched between a nearly perfect $\mathrm{Si}(111)$ substrate and a thin, incoherent, protective Si layer Kossel measurements together with a quantitative analysis yielding the position of Co atoms normal to the surface were performed.

Metallic $\mathrm{CoSi}_{2}$ layers buried in $\mathrm{Si}$ are of both fundamental and technical interest. ${ }^{12}$ Structurally, the lattice parameter of the diamond lattice of $\mathrm{Si}$ differs from the $\mathrm{CaF}_{2}$ type lattice of $\mathrm{CoSi}_{2}$ by only $1.2 \%$, providing favorable conditions for epitaxial growth. Fundamentally, a $\mathrm{CoSi}_{2}: \mathrm{Si}$ heterostructure can serve as a Schottky contact, where the height of the Schottky barrier can be explained theoretically, given a thorough knowledge of the geometrical structure of the interface. ${ }^{13,14}$

In a typical XSW experiment a plane wave, which can be considered as coming from a point source at infinite distance, is impinging on a dynamically diffracting crystal. The angle of incidence is in the vicinity of a Bragg reflection. Incident and diffracted beam interfere with each other to form a standing wave field in the region where they overlap, which extends both into the interior of the crystal and the space above the crystal. The period of the standing wave field is

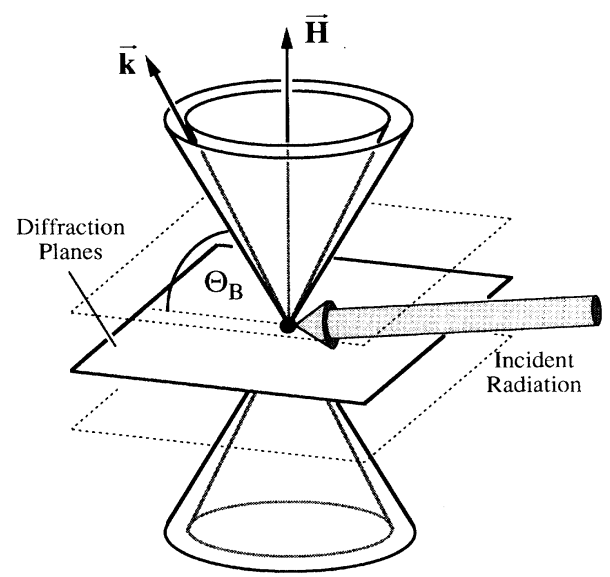

FIG. 1. Excitation of Kossel lines. Internal atoms are excited by incident radiation and subsequently deexcite emitting fluorescence. In directions $\mathbf{k}$ consistent with Bragg's law this fluorescence is diffracted by the surrounding lattice producing Kossel cones centered on reciprocal lattice vectors $\mathbf{H}$ and opening half-angle $\left(90^{\circ}-\Theta_{B}\right)$ $\left(\Theta_{B}\right.$, Bragg angle). 
equal to the lattice plane spacing and planes of constant intensity are parallel to the diffraction planes. The actual positions of the nodes and antinodes depend on the phase shift between incident and diffracted wave, which in turn depends on the angle of incidence in such a way that nodes lie on the diffraction planes for the smaller-angle side of the reflection while lying in between for the larger-angle side. Quantitatively, the relative electric field intensity of the standing wave field at a point $\Delta d / d$ within a diffraction plane spacing and at a depth $z$ below the surface can be described as a function of the angle $\Delta \Theta=\left(\Theta-\Theta_{B}\right)$ by $^{15}$

$$
\begin{aligned}
\frac{I(\Delta \Theta)}{I_{0}}= & \{1+R(\Delta \Theta)+2 P \sqrt{R(\Delta \Theta)} \\
& \left.\times \cos \left(\nu(\Delta \Theta)-2 \pi \frac{\Delta d}{d}\right)\right\} e^{-\mu_{z}(\Delta \Theta) z} .
\end{aligned}
$$

Here $R$ stands for the reflectivity, $I_{0}$ is the intensity of the incident beam and $P$ is a polarization constant. According to the dynamical diffraction theory the phase shift $\nu$ varies smoothly by $\pi \mathrm{rad}$ as the Bragg reflection is traversed. The effective attenuation coefficient $\mu_{z}(\Delta \Theta)$ includes contributions from linear absorption, primary extinction and anomalous absorption. In an actual XSW experiment the intensity $I(\Delta \Theta) / I_{0}$ is detected by measuring the fluorescence from impurity atoms at the surface or in the bulk, which in the dipole approximation is proportional to the electric field at the center of these atoms. Given an extended distribution of impurities the fluorescence yield derived from (1) becomes

$$
\begin{aligned}
Y(\Delta \Theta)= & \left\{1+R(\Delta \Theta)+2 f_{c} P \sqrt{R(\Delta \Theta)}\right. \\
& \left.\times \cos \left[\nu(\Delta \Theta)-2 \pi \Phi_{c}\right]\right\} Z(\Delta \Theta),
\end{aligned}
$$

where the coherent fraction $f_{c}$ and the coherent position $\Phi_{c}$ are the amplitude and phase of one Fourier component of the impurity distribution function. In cases where all impurity atoms lie on or near one crystallographic equivalent site, $\Phi_{c}$ marks the position of this site within the diffraction plane spacing and $f_{c}$ is the percentage of atoms occupying it. The effective thickness contributing to the yield is unity for an atomically thin surface or interface layer, and for a bulk distribution it is given by an integration of the exponential in (1) over the contributing depth.

By virtue of the optical reciprocity theorem, dynamical Kossel diffraction can be viewed as the reverse process of the standing wave mechanism described above. Applied in this context, the optical reciprocity theorem stipulates ${ }^{5,16}$ that when a monochromatic plane wave from a point source at position $B$ far away from the sample generates an electric field intensity $I$ at a point $A$ inside the sample, the same intensity will be encountered at $B$ when the source is moved to $A$ (Fig. 2).

In a standing wave experiment an incident plane wave considered to come from a point source at infinity generates an electric field intensity inside the sample which is "detected" by fluorescing impurity atoms. Moving the source to vary the incident angle $\Theta_{B}+\Delta \Theta$ results in a secondary yield as described by (2). In a Kossel diffraction experiment the impurity atoms inside or at the surface of the sample now become the point sources of radiation, excited by some ex-

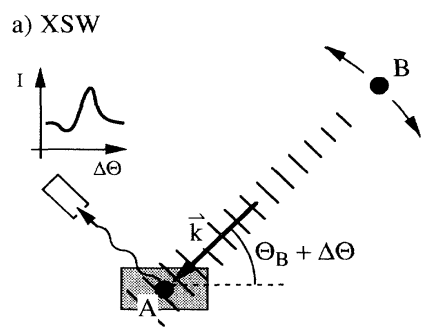

b) Kossel diffraction

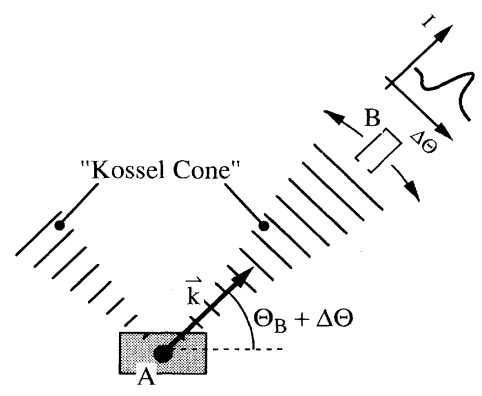

FIG. 2. (a) XSW: A source of radiation at a distant point B generates a plane wave incident on the sample under an angle $\Theta_{B}+\Delta \Theta$. The resultant electric field intensity is "detected" by fluorescing atoms at $A$. Moving the source to vary $\Delta \Theta$ produces a fluorescence yield revealing the lattice location of fluorescing atoms. (b) Kossel diffraction: Externally excited atoms at A inside the sample serve as a source of radiation which is diffracted by the surrounding lattice. The electric field intensity is measured at $\mathbf{B}$ under an observation angle $\Theta_{B}+\Delta \Theta$. Moving the detector to vary $\Delta \Theta$ results in a fluorescence yield which by virtue of the optical reciprocity theorem is equivalent to the XSW signal.

ternal beam which plays no role in further diffraction processes since it differs in energy from the fluorescence energy. The electric field intensity produced in all of space by the internal source is then measured at infinity, which for practical purposes is achieved by using a highly collimating analyzer crystal combined with a fluorescence detector. Moving this kind of angularly resolving detector to vary the angle of observation $\Theta_{B}+\Delta \Theta$ completes the reversal of the standing wave scheme and must then, by application of the optical reciprocity theorem, result in the very same secondary yield as encountered before with standing waves. It should be mentioned, however, that regular XSW measurements are performed with a $\sigma$-polarized incident beam, whereas Kossel diffracted fluorescence is unpolarized. Therefore, the singlepolarization yield given in (2) has to be summed over $\sigma$ and $\pi$ polarizations in the Kossel case.

The argumentation given above for the equivalence of $\mathrm{x}$-ray standing waves and Kossel diffraction is strikingly simple and intuitive, if one accepts the applicability of the reciprocity theorem in this case. However, the fluorescence yield as given in (2) can also be obtained "directly" for the case of Kossel diffraction, as was shown by Hutton, Trammell, and Hannon. ${ }^{8}$

A practical experimental configuration for recording angularly resolved Kossel lines with the aim to determine lattice locations of highly diluted bulk impurities or atomically 


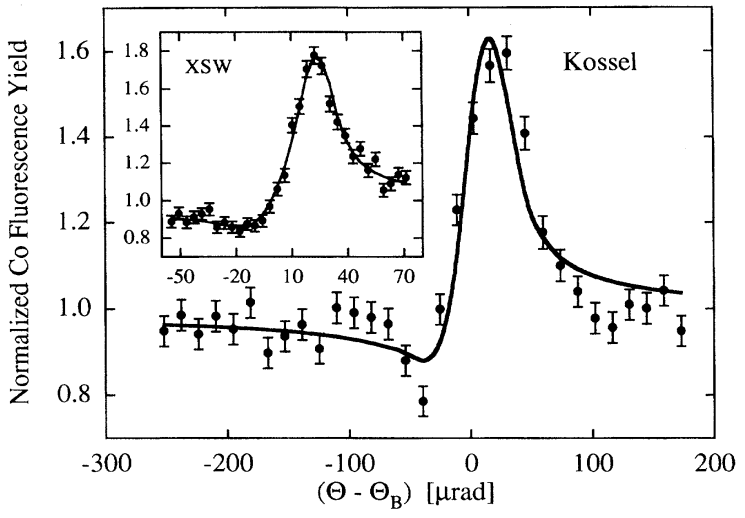

FIG. 3. Normalized fluorescence data and theoretical fits from a Kossel diffraction (main graph) and corresponding XSW measurement (inset). The sample consists of an atomically thin, buried $\mathrm{CoSi}_{2}$ layer on a $\mathrm{Si}(111)$ substrate. Extracted coherent positions for Co atoms are $\Phi_{c}=0.903 \pm 0.01$ and $\Phi_{c}=0.896 \pm 0.01$, respectively, with coherent fractions of $f_{c}=0.47 \pm 0.02$ and $f_{c}=0.483 \pm 0.01$.

thin surface layers in nearly perfect crystals was implemented at the undulator beamline BW1 (Ref. 17) of HASYLAB and is described elsewhere. ${ }^{11}$

A "pink" undulator x-ray beam impinges on the sample exciting fluorescence which is diffracted within the sample and recorded by a combination of analyzer crystal and $\mathrm{Si}(\mathrm{Li})$ detector. By virtue of its narrow angular acceptance the analyzer can be thought of as being located very far from the sample.

A special concern in working with low concentrations of fluorescing atoms is the stability of the relative orientation between sample and analyzer crystals over long periods of data accumulation time. To circumvent any problem of uncontrolled drifts a scheme of dynamical stabilization was adopted. The sample is set at such an angle with the incident synchrotron radiation beam that a Bragg-diffracted reference beam at an energy slightly different from the Kossel fluorescence line is generated and detected in an ion chamber after passing the analyzer. The analyzer is then repeatedly rocked back and forth through the reflectivity curve, which is evaluated in terms of angular position so that slow drifts in relative orientation can be offset by the controlling computer from scan to scan.

A $7 \AA$ thick, buried layer of $\mathrm{CoSi}_{2}$ on a $\mathrm{Si}(111)$ substrate, capped with a thin, protective, incoherent layer of Si served as a sample in this current study accompanied by a matching $\mathrm{Si}(111)$ analyzer. Co $\mathrm{K} \alpha$. fluorescence from atoms of the buried $\mathrm{CoSi}_{2}$ layer is radiated both into the space above the sample, creating a constant fluorescence background, and also into the nearly perfect substrate, where the Kossel lines are formed. One half of the (111) Kossel cone is directed back into the space above the sample and can be recorded there as a function of relative sample-to-analyzer orientation. Results from such a measurement together with a theoretical least-squares fit are shown in the main graph of Fig. 3. For comparison the inset shows a regular XSW measurement of the same sample. Both measurements are fully consistent with each other in yielding independently coherent positions for Co atoms relative to the $\mathrm{Si}$ substrate along the (111) direction of $\Phi_{c}=0.903 \pm 0.01$ and $\Phi_{c}=0.896 \pm 0.01$, respectively, together with coherent fractions of $f_{c}=0.47 \pm 0.02$ and $f_{c}=0.483 \pm 0.01$. The Kossel signal is slightly noisier than the XSW signal owing to the incident "pink" beam with its associated background problems, which could be reduced by using a broad band pass monochromator. The differing widths of Kossel and XSW fluorescence yields are due to the difference in energy between Kossel fluorescence at $6.9 \mathrm{keV}$ and the XSW energy tuned to $8.9 \mathrm{keV}$ and from a difference in asymmetry of Kossel analyzer and XSW monochromator. The influence of the Kossel analyzer on the instrumental resolution is taken into account by modeling the reflectivity of the Bragg-diffracted reference beam in much the same way as XSW rocking curves are used to characterize the monochromator transfer function in XSW measurement. Adverse effects from probing a conical Kossel wave field with a planar analyzer crystal are minimized in the experimental set-up by placing a narrow slit behind the analyzer that restricts the beam perpendicular to the diffraction plane of the analyzer. Thereby only a thin slice of the Kossel cone is permitted to enter the detector. An additional broadening of Kossel lines stems from the inherent mixing of $\sigma$ and $\pi$ polarizations, which usually is suppressed in XSW when using mostly $\sigma$-polarized synchrotron radiation in the plane of the source's orbit. After including this polarization mixing explicitly in the model function underlying the least-squares fits, the shape of the yields and the consistency of coherent positions and coherent fractions extracted clearly demonstrate the equivalence of Kossel diffraction and XSW and proves the practicability of the Kossel approach in terms of a structure analysis analogous to what is achieved by regular XSW.

Concerning the geometrical structure of the $\mathrm{CoSi}_{2}: \mathrm{Si}$ interface two major models have been discussed in earlier investigations. ${ }^{13,14}$ The first one assumes bonding between $\mathrm{Co}$ and $\mathrm{Si}$ atoms across the interface, whereas the alternative model is based on Si-Si bonds. Discounting any relaxation, the first model would imply a coherent Co position of 2.734 $\AA$ while the second would place these atoms at $3.514 \AA$ with respect to $\mathrm{Si}(111)$ diffraction planes. Comparing these theoretical values with the coherent position obtained in the current Kossel measurements of $(0.90 \pm 0.01) d_{\mathrm{Si}(111)}$ $=(2.82 \pm 0.03) \AA$ one is led to conclude that for a buried $\mathrm{CoSi}_{2}$ layer with a thickness of $7 \AA$ the model with the Co-Si bond is realized together with a relaxation of interface bonds by $(0.09 \pm 0.03) \AA$. This result is consistent with outcomes of previous studies. ${ }^{13,14}$

In conclusion, it was demonstrated that angularly resolved Kossel lines inherently contain the same information about the position and the order of the fluorescing sources as yields from regular XSW measurements do and that Kossel diffraction studies are practical even for samples with very low concentration of impurities or overlayer atoms. For an atomical thin, buried layer of $\mathrm{CoSi}_{2}$ on a $\mathrm{Si}(111)$ substrate Kossel measurements could be analyzed quantitatively to identify the model with $\mathrm{Co}-\mathrm{Si}$ bonds forming the $\mathrm{CoSi}_{2}: \mathrm{Si}$ interface and to determine their bond relaxation.

However, some principal differences between Kossel diffraction and regular XSW have to be mentioned. While the use of synchrotron radiation permits to select just one polarization component $(\sigma)$ in regular XSW experiments, fluores- 
cence from internal sources is always unpolarized and leads to an angular broadening of the fluorescence yield.

In addition, for dispersive set-ups, the amount of signal distortion can be reduced by decreasing the divergence of the incident $\mathrm{x}$-ray beam in XSW measurements. Especially at synchrotron radiation sources of the third generation, the high brilliance is expected to greatly reduce adverse effects of dispersiveness. ${ }^{18}$ For Kossel diffraction the source divergence is determined by the natural linewidth of the fluorescence, thus the resolution can only be improved with a more complicated analyzer system. Nevertheless, the Kossel scheme appears to be particularly attractive for the fact that several Kossel lines are generated and can be recorded simultaneously, each carrying a positional information along the direction of its associated $\mathbf{H}$ vector. Consequently full three-dimensional information about lattice locations is accessible in a single experiment. Also, since Kossel lines can be excited with other sources (electrons, protons, ions, ... .), the Kossel diffraction method described above might be well suited for laboratory-based measurements.

We would like to thank C. Schwarz and H. von Känel for preparing the $\mathrm{CoSi}_{2}: \mathrm{Si}$ samples.
${ }^{1}$ A. Szöke, in Short Wavelength Coherent Radiation: Generation and Applications, Proceedings of the Topical Meeting, edited by T. Attwood and J. Boker, AIP Conf. Proc. No. 147 (AIP, New York, 1986).

${ }^{2}$ M. Tegze and G. Faigel, Europhys. Lett. 16, 41 (1991).

${ }^{3}$ W. Kossel, V. Loeck, and H. Voges, Z. Phys. 94, 139 (1935).

${ }^{4}$ M. v. Laue, Röntgenstrahlinterferenzen (Akademische Verlagsgesellschaft, Frankfurt, Germany, 1960).

${ }^{5}$ W. Schülke and O. Brümmer, Z. Naturforsch. Teil A 17, 208 (1962).

${ }^{6}$ G. Nolze and V. Geist, Cryst. Res. Technol. 27, 421 (1992).

${ }^{7}$ J. M. Cowley, Acta Crystallogr. 17, 33 (1964).

${ }^{8}$ J. T. Hutton, G. T. Trammell, and J. P. Hannon, Phys. Rev. B 31, 743 (1985).

${ }^{9}$ D. Stephan, W. Blau, H. J. Ullrich, and G. E. R. Schulze, Krist. Tech. 9, 707 (1974); 11, 475 (1976).
${ }^{10}$ T. Takahashi and M. Takahasi, Jpn. J. Appl. Phys. 32, 5159 (1993).

${ }^{11}$ Th. Gog, D. Novikov, J. Falta, A. Hille, and G. Materlik, J. Phys. IV (France) 4, C9-449 (1994).

${ }^{12}$ S. Mantl, Mater. Sci. Reports 8, 1 (1992).

${ }^{13}$ A. e. M. J. Fischer, E. Vlieg, J. F. van der Veen, M. Clausnitzer, and G. Materlik, Phys. Rev. B 36, 4769 (1987).

${ }^{14}$ J. Zegenhagen, K.-H. Huang, B. D. Hunt, and L. J. Schowalter, Appl. Phys. Lett. 51, 1176 (1987).

${ }^{15}$ M. J. Bedzyk and G. Materlik, Phys. Rev. B 32, 6456 (1985).

${ }^{16}$ C. v. Fragstein, Optik (Stuttgart) 11, 301 (1954).

${ }^{17}$ R. Frahm, J. Weigelt, G. Meyer, and G. Materlik, Rev. Scientific Instrum. (to be published).

${ }^{18}$ T. Gog, A. Hille, D. Bahr, and G. Materlik, Rev. Scientific Instrum. (to be published). 